\title{
Physiologie des bactéries du genre Lactococcus en conditions de privation nutritionnelle. Une revue
}

\author{
C Foucaud, D Hemme \\ INRA, Station de recherches laitières, 78350 Jouy-en-Josas, France
}

(Reçu le 28 novembre 1989; accepté le 30 janvier1990)

\begin{abstract}
Résumé - Les bactéries du genre Lactococcus placées en conditions de privation nutritionnelle présentent une viabilité faible, susceptible d'être influencée par des facteurs liés aux souches étudiées et aux conditions de culture ou de privation nutritionnelle. La dégradation des constituants cellulaires concerne les glucides, les lipides, les protéines, les acides aminés et les acides nucléiques. II n'est pas possible d'associer la dégradation sélective d'un constituant à la perte de viabilité des cellules. La libération des produits de dégradation (protéines, acides aminés) et de certaines enzymes intracellulaires ( $\beta$-galactosidase, lactate déshydrogénase, prolyliminopeptidase) dépend de la rupture de la paroi qui met en cause une ou des autolysine(s), à ce jour encore peu connue(s). Les modifications métaboliques associées aux conditions de privation nutritionnelle concernent surtout la régulation du métabolisme glucidique obtenue par la modulation des activités enzymatiques de la glycolyse en réponse aux fluctuations de la concentration des effecteurs intracellulaires. La perte de plasmides en conditions de privation nutritionnelle est également rapportée sans pour autant que le mécanisme soit connu.
\end{abstract}

Lactococcus / privation nutritionnelle / viabilité / composition intracellulaire / rejet de matériel / métabolisme / plasmide / autolysine

Summary - Physiology of Lactococcus bacteria during starvation. Starved cells of genus Lactococcus have a low survival rate which is dependent upon the strains used, culture conditions and the starvation regime. Cellular components that are degraded are carbohydrates, lipids, proteins, aminoacids and nucleic acids. The degradation of a particular component could not be associated directly to loss of cell viability. The release of degradation products (proteins, aminoacids) and of some intracellular enzymes ( $\beta$-galactosidase, lactate dehydrogenase or prolyliminopeptidase) depends on the rupture of the cell wall which implicates one or more autolysin(s) which are still poorly described. The modifications of the overall cell metabolism during starvation essentially concerned the regulation of carbohydrates metabolism with the enzymes involved in glycolysis brought about by changes in intracellular effector levels. The loss of plasmids during starvation is also reported but the mechanism by which this loss occurred still remains unknown.

Lactococcus / starvation / survival / intracellular composition / release / metabolism / plasmid / autolysin 


\section{INTRODUCTION}

Les bactéries lactiques sont des bactéries Gram+$^{+}$, asporogènes formant des chaînes et qui dégradent le lactose en acide lactique rejeté dans le milieu extérieur.

L'étude de la physiologie des bactéries lactiques en conditions de privation nutritionnelle fait appel à plusieurs types d'expériences :

- suspension des cellules en solution saline tamponnée à la température de croissance;

- incubation prolongée dans le milieu de culture après arrêt de la croissance dû à l'épuisement d'un nutriment essentiel;

- croissance en culture continue à un taux suffisamment faible pour que la mortalité bactérienne spontanée contribue à la dynamique d'un système à l'état stable.

Dans cette revue, nous nous limiterons au genre Lactococcus qui a souvent servi de modèle d'études et qui comprend, par ailleurs, les souches les plus utilisées en fromagerie dans le monde anglo-saxon.

D'importantes études relatives à la physiologie des lactocoques en conditions de privation nutritionnelle ont été publiées en 1968 et 1969 par Thomas, Batt et leurs collaborateurs en Nouvelle-Zélande. Les travaux dans ce domaine ont été ensuite épisodiques avant de reprendre récemment (Vegarud et Langsrud, 1987; Niskasaari, 1989; notre laboratoire depuis 1987).

Cette recrudescence d'intérêt pour la physiologie en conditions de privation nutritionnelle de ce genre très important dans l'industrie laitière se justifie, en grande partie par les mécanismes physiologiques impliqués, notamment les phénomènes naturels de lyse avec libération d'enzymes intracellulaires dans le milieu extérieur.
En effet, au cours de la formation du caillé ou de l'affinage des fromages (Cogan et Daly, 1987; Law, 1987), certaines des enzymes impliquées, protéases et peptidases (Thomas et Pritchard, 1987), ne sont généralement mises au contact de leur substrat qu'au moment de l'autolyse bactérienne (Ohmiya et Sato, 1970; Law et al, 1974). Les conditions dans lesquelles se produit cette lyse sont donc importantes pour le déroulement du processus d'affinage.

D'autre part, en état de cellules non proliférantes ou lors du processus d'autolyse, les cellules de lactocoques sont susceptibles de libérer des composés cellulaires qui peuvent agir sur le milieu environnant, sur les autres bactéries du levain ou permettre la croissance d'une microflore secondaire du fromage (Thomas, 1987).

Ces cellules peuvent, par ailleurs, métaboliser certains composés du milieu en les prélevant et en excrétant les produits formés.

Enfin, leur métabolisme peut être modifié. Par exemple, la dégradation du lactose par voie hétérofermentaire mène à la production de métabolites autres que le lactate qui peuvent être impliqués dans l'arôme des fromages.

Dans ce contexte, les études relatives à la physiologie des bactéries du genre Lactococcus en conditions de privation nutritionnelle tendent à :

- compléter des connaissances fondamentales relatives à la physiologie de ces bactéries, notamment leurs capacités d'adaptation ou de régulation et leurs potentialités dans ces conditions puisqu'elles sont surtout connues en conditions optimales;

- apprécier les phénomènes survenant dans ces conditions, par exemple la dégradation et la perte de composés cellulaires, 
pour mieux connaître et prévoir ce qui est susceptible de se passer dans le milieu naturel;

- cerner les facteurs qui influencent le comportement des cellules afin de mieux les maîtriser ou les utiliser;

- obtenir des données sur l'(les) autolysine(s) pour mieux comprendre la lyse cellulaire survenant dans le fromage et ses mécanismes;

- mettre en évidence des souches dont le comportement physiologique particulier pourrait être exploité, par exemple une capacité lytique ou catabolique importante ou au contraire limitée.

Dans cette revue, nous considérerons successivement la viabilité des bactéries du genre Lactococcus puis les modifications structurales, morphologiques, métaboliques ou génotypiques survenant au cours de la privation nutritionnelle et les facteurs qui les conditionnent lorsqu'ils sont connus.

\section{Étude de la viabilité bactérienne}

Postgate (1976) donne une définition pragmatique de la viabilité des procaryotes: la viabilité d'une population microbienne est la propriété que possèdent les individus de cette population capables de se multiplier lorsque leur sont offertes des conditions suffisantes pour la croissance.

Ainsi, le terme "viable" s'applique aux cellules capables de se multiplier et de former des colonies et le terme "vivant" est réservé aux cellules montrant d'autres signes de viabilité, tels qu'une activité métabolique, même si ces cellules sont incapables de se diviser dans des conditions optimales (Mason et al, 1986; Roznak et Colwell, 1987).

Toutefois, la capacité des cellules à croître et à se multiplier est le plus souvent le seul critère utilisé pour différencier ces organismes.

Ainsi, au cours des études relatives à la viabilité des cellules de Lactococcus lactis, la technique de numération sur milieu gélosé, après incubation ou selon la méthode de Postgate et al (1961) basée sur une incubation de courte durée sur une pellicule de gélose suivie d'un comptage différentiel au microscope, a été couramment mise en ceuvre.

Cette méthodologie est critiquée notamment parce que les cellules de Lc lactis se présentent sour forme de chaînes plus ou moins longues. Thomas et al (1969), au terme d'observations microscopiques, concluent que les cellules de Lc lactis ML3 ont tendance à s'agréger lors d'un séjour prolongé en solution tampon phosphate. Ceci explique la diminution dans le temps du nombre total de chaînes, mais il n'y a pas de réduction significative du nombre total de cocci au cours de la privation nutritionnelle. Afin de pallier cette ambiguilté, I'utilisation d'un broyeur de type UltraTurrax, préconisée par Martley (1972), a parfois été réalisée pour homogénéiser la longueur moyenne des chaînes mais uniquement lors de la mise en suspension des cellules dans la solution tampon (Niskasaari, 1989).

Lorsque des cellules de Lc lactis ML3 sont transférées en solution tampon phosphate, la viabilité cellulaire, estimée par la méthode de Postgate et al (1961), décline selon une courbe sigmoïde. La viabilité cellulaire est de $50 \%$ de sa valeur initiale après $8 \mathrm{~h}$ de séjour à $30^{\circ} \mathrm{C}$ en solution tampon phosphate additionnée d'acide éthylène diamine tétraacétique (EDTA) (Thomas et Batt, 1968).

L'observation de ces organismes en microscopie électronique révèle des modifications structurales progressives des cellules (Thomas et al, 1969; Ohmiya et Sato, 
1970). Une dislocation du matériel cellulaire survient très tôt, les ribosomes disparaissent tandis que le pourcentage de cellules lysées augmente suite à une rupture conjointe de la paroi et de la membrane cellulaire.

\section{Influence du stade de croissance des cellules}

Thomas et Batt (1968) rapportent que la viabilité bactérienne de la souche ML3 n'est pas influencée par le stade de croissance auquel sont prélevées les cellules provenant d'un milieu limité en lactose. Dans le cas d'Aerobacter aerogenes, Postgate et Hunter (1962) suggèrent que, pour une population génétiquement uniforme, la phase de croissance a une faible influence sur les caractéristiques de la viabilité bactérienne au cours de la privation nutritionnelle.

Cependant, des études similaires ont montré, notamment chez Lc lactis subsp cremoris (Mou et al, 1976; Niskasaari, 1989), L c lactis subsp lactis (Foucaud et al, soumis pour publication) que l'activité autolytique des souches augmente au cours de la phase exponentielle de croissance pour commencer à diminuer peu avant la transition vers la phase stationnaire. Cette observation renforce l'hypothèse selon laquelle les autolysines ont un rôle important au cours de la croissance et de la division cellulaire (Young, 1966).

\section{Influence des caractères des souches}

L'étude de la physiologie des lactocoques en conditions de privation nutritionnelle a permis de mettre en évidence un comportement globalement similaire mais plus ou moins accentué selon les souches (Langsrud et al, 1978; Vegarud et al, 1983; Vegarud et Langsrud, 1987; Niskasaari, 1989). La susceptibilité des organismes et l'importance de l'autolyse bactérienne ont été reliées à certains caractères des souches.

Lors de leur culture en milieu liquide ou de leur séjour en solution tampon, les cellules de Lc lactis qui développent de longues chaînes, montrent des activités autolytiques réduites (McDonald, 1971; Foucaud et al, soumis pour publication). Ceci souligne encore le rôle du système enzymatique responsable de l'autolyse bactérienne dans la division et la croissance cellulaire (Young, 1966). Des différences dans le comportement des souches peuvent également être associées à des compositions différentes de la paroi cellulaire et de la structure périphérique (Sundman, 1957; Kontusaari et Forsén, 1987) ou être dues à une modification des systèmes autolytiques et/ou à des différences dans leur régulation par l'acide téïchoique associé à la membrane et par les polymères liés au peptidoglycane (Niskasaari, 1989).

\section{Influence de la source de carbone présente dans le milieu de culture}

L'importance de la source de carbone présente dans le milieu de culture des souches sur la susceptibilité des bactéries à la lyse a été observée dans le cas de $L c$ lactis (Moustafa et Collins, 1968; Vegarud et al, 1983). Les bactéries ne peuvent utiliser le glucose comme précurseur adéquat de la galactosamine présente dans la paroi cellulaire (Moustafa et Collins, 1968; Prasad et Freese, 1974). La phosphoglucomutase, concernée par cette réaction, est paradoxalement sensible à des températures proches de $30{ }^{\circ} \mathrm{C}$, c'est-à-dire de l'optimum de croissance des cellules. 
Influence de la nature de la solution tampon

Aucun travail n'a réellement porté sur l'influence de la nature du tampon employé pour la suspension de cellules de Lc lactis. La solution utilisée est généralement du tampon phosphate de sodium et/ou de potassium (Moustafa et Collins, 1968; Thomas et Batt, 1968; Ohmiya et Sato, 1969; Krishna et Dutta, 1976; Mou et al, 1976; Niskasaari, 1989), parfois du tampon phosphate/citrate de sodium (Thomas et Batt, 1968). Nous avons testé la viabilité des cellules de $L c$ lactis subsp lactis biovar diacetylactis CNRZ 125 dans plusieurs solutions tampon 50 millimolaires à $\mathrm{pH} 6$. Après $30 \mathrm{~h}$ de séjour, la meilleure viabilité des cellules est obtenue avec le citrate de sodium $(95 \%)$ alors qu'elle n'est pas que de $80 \%$ avec le tampon pipérazine $\mathrm{N}-\mathrm{N}$ bis-[2-acide sulfonique] (PIPES), de $66 \%$ avec le tampon succinate de sodium et de $25 \%$ avec le tampon phosphate de potassium (Foucaud et al, soumis pour publication).

Quelles que soient les études, le choix de la solution tampon a rarement été justifié si ce n'est a posteriori parce qu'elle favorise le phénomène étudié. II ressort cependant de quelques travaux que les anions citrate (Elmros et al, 1976) et succinate (Bridson et Brecker, 1970) peuvent se comporter comme des agents chélatants faibles, capables de complexer certains ions dont le $\mathrm{Ca}^{2+}$ et le $\mathrm{Mg}^{2+}$. Ito et al (1987) ont montré le rôle du citrate dans l'inhibition de l'activité des enzymes lytiques chez les streptocoques du groupe $\mathrm{N}$.

\section{Influence du pH de la solution tampon}

L'activité autolytique des cellules de $L c$ lactis subsp lactis souche DRC1 (Moustafa et Collins, 1968) et Lc lactis subsp cremoris
(Mou et al, 1976), évaluée par mesure de la turbidité des suspensions, est optimale pour des valeurs de $\mathrm{pH}$ comprises entre 6 et 7 . Elle est moindre pour des valeurs de $\mathrm{pH}$ plus faibles ou plus fortes probablement à cause de la dénaturation de la ou des autolysine(s) comme le suggèrent Tipper (1969) et Cornett et al (1979) pour Staphylococcus aureus et Streptococcus faecium.

La viabilité des cellules de Lc lactis subsp lactis ML3, estimée par comptage des cellules viables, est maximale pour des valeurs de $\mathrm{pH}$ proches de 7 (Thomas et Batt, 1968, 1969c). Elle décroít nettement lorsque les valeurs de $\mathrm{pH}$ diminuent et, à pH 4, la viabilité des cellules diminue de $50 \%$ en $30 \mathrm{~min}$.

Les résultats des rares études relatives à l'influence du $\mathrm{pH}$ divergent nettement et ne permettent pas actuellement de conclure.

\section{Influence de la concentration de la solution tampon}

Les concentrations permettant une viabilité optimale sont comprises entre 50 et 750 $\mathrm{mmol} / \mathrm{l}$ (Thomas et Batt, 1968; Mou et al, 1976; Vegarud et Langsrud, 1982). Une concentration saline supérieure offrirait aux cellules une protection osmotique contre la lyse (Hinks et al, 1978).

\section{Influence de la température d'incubation}

La viabilité augmente nettement lorsque la température d'incubation des suspensions diminue de $45^{\circ} \mathrm{C}$ à $3{ }^{\circ} \mathrm{C}$ (Thomas et Batt, 1968). Des résultats semblables ont été rapportés pour des souches de $L c$ lactis subsp cremoris (Mou et al, 1976). Une 
température d'incubation supérieure à $45^{\circ} \mathrm{C}$ conduit à une inactivation complète du système autolytique bactérien.

\section{Influence de l'agitation et de l'atmosphère}

Une agitation ménagée produite à l'aide d'un barreau magnétique n'a pas d'effet mesurable sur les courbes de viabilité de Lc lactis ML3 (Thomas et Batt, 1968). Une agitation plus vigoureuse conduit à une diminution de la viabilité $(50 \%$ de réduction en 2-3 h).

L'air comme l'azote diminue la viabilité des cellules lorsque des bulles de ces gaz traversent la suspension $(50 \%$ de diminution en 4-5 $\mathrm{h}$ pour l'air et en 2-3 $\mathrm{h}$ pour l'azote). Cette diminution en condition anaérobie n'a guère été expliquée si ce n'est en avançant l'hypothèse que certaines valeurs de potentiel d'oxydoréduction seraient nécessaires à la viabilité (Thomas et Batt, 1968).

\section{Influence de l'addition de divers composés}

\section{EDTA}

De faibles concentrations d'EDTA (10 $\mu \mathrm{mol} / \mathrm{l})$ n'ont qu'un très léger effet sur la viabilité des cellules de Lc lactis placées en solution tampon phosphate alors que des concentrations élevées $(10 \mathrm{mmol} / \mathrm{l}) \mathrm{di}$ minuent la viabilité (Thomas et Batt, 1968) probablement à cause de la capacité de cet agent à déstabiliser la paroi cellulaire (Gray et Wilkinson, 1965; Asbell et Eagon, 1966) ou les ribosomes (Wade, 1961) par chélation des cations impliqués dans cette structure.

\section{lons divalents}

L'addition de $\mathrm{Hg}^{2}+(10 \mu \mathrm{mol} / \mathrm{l})$ à la solution de tampon phosphate entraîne la mort de toutes les cellules de Lc lactis ML3 après 5 min de séjour (Thomas et Batt, 1968). Notons que $\mathrm{HgCl}_{2}$ a été utilisé comme conservateur dans le lait cru. A notre connaissance, aucune information n'est toutefois disponible concernant le rôle de cet ion chez les bactéries lactiques.

Dans les mêmes conditions, la toxicité des ions testés décroît selon l'ordre $\mathrm{Cu}^{2+}$, $\mathrm{Ni}^{2+}, \mathrm{Fe}^{2+}, \mathrm{Co}^{2+}, \mathrm{Zn}^{2+}, \mathrm{Pb}^{2+}$. Les ions $\mathrm{Ca}^{2+}, \mathrm{Mn}^{2+}$ et $\mathrm{Sn}^{2+}$ sont sans effet sur la viabilité cellulaire. Des études semblables, réalisées pour d'autres genres bactériens, ont mis en œuvre des concentrations ioniques plus élevées, de 1 à $100 \mathrm{mmol} / \mathrm{l}$ contre $10 \mu \mathrm{mol} / \mathrm{l}$, ce qui pourrait expliquer l'inhibition de l'autolyse bactérienne, rapportée pour Lb helveticus, par les ions $\mathrm{Ca}^{2+}$ (Bie et Sjöström, 1975) ou par les ions $\mathrm{Ca}^{2+}$ et $\mathrm{Mn}^{2+}$ pour Bacillus subtilis (Svarachorn, 1989).

L'addition de $\mathrm{Mg}^{2+}$ dans la solution tampon phosphate augmente considérablement la viabilité des cellules de $L c$ lactis ML3 (Thomas et Batt, 1968). Une viabilité de $50 \%$ est obtenue après 29 h de séjour en solution tampon additionnée de $\mathrm{Mg}^{2+}$ ( $1 \mathrm{mmol} / \mathrm{l}$ ) contre $8 \mathrm{~h}$ en absence de $\mathrm{Mg}^{2+}$. Les expériences décrites par McLeod et Snell (1950) chez Lb arabinosus et par Abelson et Aldous (1950) chez E coli montrent 2 effets distincts des ions magnésium : d'une part un effet protecteur contre la toxicité du cuivre que Thomas et Batt (1968) détectent en qualité d'impureté dans la solution tampon à une concentration faible $(1 \mu \mathrm{mol} / \mathrm{l})$ et d'autre part un effet stabilisant pour les ribosomes. Thomas et Batt (1969a) montrent ainsi la réduction de l'excrétion des pentoses et des substances absorbant dans l'UV. L'effet protecteur des 
ions $\mathrm{Mg}^{2+}$ se confirme lors de l'utilisation de populations bactériennes denses susceptibles de libérer cet ion dans l'environnement à une concentration stabilisante (Thomas et Batt, 1968).

Une réduction de l'autolyse bactérienne, corrélée à une augmentation de la concentration en $\mathrm{NaCl}$ (0 à 4,5\%) est observée pour la plupart des souches de $L c$ lactis testées (Bie et Sjöström, 1975; Vegarud et al, 1983). Le manque de sensibilité d'une souche de $L$ c lactis subsp cremoris en culture (Bie et Sjöström, 1975) pourrait être intéressante si l'on considère l'influence du salage sur le processus de maturation des fromages (Guinee et Fox, 1987).

\section{Inhibiteurs du métabolisme}

L'addition d'acide iodoacétique (10 $\mu \mathrm{mol} / \mathrm{l})$ diminue considérablement la viabilité des cellules de $L$ c lactis ML3 placées en solution tampon phosphate : $50 \%$ de perte de viabilité en $1 \mathrm{~h}$ (Thomas et Batt, 1968).

L'effet bactéricide du bleu de méthylène dépend de sa concentration. Une concentration supérieure à $20 \mu \mathrm{mol} / \mathrm{l}$ montre un effet immédiat alors qu'avec $1 \mu \mathrm{mol} / \mathrm{l}$, la viabilité est supérieure $(50 \%$ après 6 à $7 \mathrm{~h}$ de séjour en tampon phosphate).

L'addition d'arséniate ou de fluorure de sodium produit également une légère diminution de la viabilité. Le cyanure de potassium, le malonate de sodium, l'azide de sodium et le 2,4-dinitrophénol sont sans effet.

\section{Spermine}

Certaines diamines aliphatiques, dont la spermine, ont un effet stabilisant marqué sur les protoplastes et les bactéries osmotiquement sensibles (Mager, 1959; Tabor, 1962) en réduisant la perte de matériel ab- sorbant dans I'UV et la lyse cellulaire chez les bactéries lactiques (Moustafa et Collins, 1968).

\section{Substrats}

\section{Glucides}

L'addition de glucose, galactose, fructose ou lactose $(10 \mathrm{mmol} / \mathrm{l})$ à une suspension de Lc lactis ML3 placée en solution tampon phosphate, diminue la viabilité des organismes d'un facteur voisin de 6 , quel que soit le stade de croissance auquel ont été prélevés les organismes ou l'élément limitant leur croissance (Thomas et Batt, $1968,1969 c)$. Cette réduction de la viabilité n'est pas due à l'effet toxique de l'acide lactique produit (Thomas et Batt, 1968). L'addition de $\mathrm{Mg}^{2+}$ restaure un taux de viabilité comparable à celui d'un système témoin dépourvu de glucose et de $\mathrm{Mg}^{2+}$ (Thomas et Batt, 1968) ou augmente la viabilité des organismes en présence de glucose (Thomas et Batt, 1969c).

La synthèse d'ARN et de protéines par les cellules de Lc lactis ML3 placées en conditions de privation nutritionnelle a été mise en évidence lors de l'addition de glucose sans que l'on puisse assurer que cela favorise la viabilité des cellules (Thomas et Batt, 1969b, 1969c). Dans ces conditions, le métabolisme du glucose $(10 \mathrm{mmol} / \mathrm{l})$ est un phénomène linéaire et représente la formation de 17,7 mmol ATP par $\mathrm{g}$ de poids sec et par $h$, soit 4 fois moins qu'au cours de la croissance (Thomas et Batt, 1969c).

Lorsque le glucose est fourni continuellement aux organismes placés en solution tampon phosphate additionnée de $\mathrm{Mg}^{2+}$, la viabilité est liée au taux d'addition du glucose. Avec des apports faibles (250 et 500 $\mu \mathrm{mol}$ par $\mathrm{g}$ de poids sec et par $\mathrm{h}$ ), la viabilité augmente d'un facteur 4 . Ceci correspond à un besoin énergétique de mainte- 
nance de 45 à $90 \mathrm{mg}$ de glucose par $\mathrm{g}$ de poids sec et par $h$ qui est du même ordre que celui des bactéries aérobies (Pirt, 1965).

\section{Acides aminés}

En présence de $\mathrm{Mg}^{2+}$, l'addition soit d'alanine, d'acides aspartique et glutamique (10 mmol/l) qui constituent la majeure partie des acides aminés libres des cellules, soit d'acides aminés d'un hydrolysat de caséine $(0,5 \%)$ augmente la viabilité des cellules de $L$ c lactis ML3 (50\% de viabilité après $38 \mathrm{~h}$ ou $50 \mathrm{~h}$, respectivement) (Thomas et Batt, 1968). Les acides aminés prolongent probablement la viabilité de $L c$ lactis en fournissant une source limitée d'énergie pour la maintenance des cellules et des acides aminés pour le turn-over des cellules, ce qui minimiserait la dégradation des composés essentiels. L'addition d'arginine $(10 \mathrm{mmol} / \mathrm{l})$ réduit la viabilité des cellules de $L c$ lactis ML3 placées en conditions de privation nutritionnelle dans les mêmes proportions que l'addition de glucose, soit une perte de $50 \%$ de viabilité en $2,5 \mathrm{~h}$ (Thomas et Batt, 1969c). L'addition de $\mathrm{Mg}^{2+}$ supprime ce phénomène pour prolonger la viabilité des cellules qui est de $50 \%$ après $55 \mathrm{~h}$ (Thomas et Batt, 1968, 1969c).

La synthèse d'ARN et de protéines est également mise en évidence en présence d'arginine (Thomas et Batt, 1969c). Cette synthèse est toutefois moins importante qu'en présence de glucose car la production d'ATP à partir de l'arginine est 7,5 fois moindre (Thomas et Batt, 1969c). Les bactéries placées en conditions de privation nutritionnelle sont extrêmement sensibles au $\mathrm{pH}$ externe, mais l'addition d'arginine en présence de $\mathrm{Mg}^{2+}$ augmente la viabilité de $L$ c lactis, quelle que soit la valeur de pH comprise entre 4 et 8 (Thomas et Batt, 1969c). Pour des $\mathrm{pH}$ acides, cet effet peut être dû à l'action neutralisante de l'ammoniaque produit par dégradation de l'arginine (Thomas et Batt, 1969c). Ce résultat renforce I'hypothèse de Dawes et Ribbons (1962, 1964) selon laquelle le contrôle du $\mathrm{pH}$ interne est une des fonctions du métabolisme endogène. Poolman et al (1987a) ont montré par ailleurs que les cellules de L c lactis ML3, métabolisant l'arginine pour la synthèse de l'ATP entre $\mathrm{pH} 5$ et 7 , maintiennent un $\mathrm{pH}$ intracellulaire proche de 7 c'est-à-dire 0,3 à 0,4 unité $\mathrm{pH}$ plus bas que les cellules métabolisant le lactose dans les mêmes conditions.

L'addition de vitamines, purines et pyrimidines en présence de $\mathrm{Mg}^{2+}(1 \mathrm{mmol} / \mathrm{l})$ ou d'acétate de sodium (10 mmol/l) n'a pas d'effet sur la viabilité des cellules de $L c$ lactis ML3 en solution tampon (Thomas et Batt, 1968).

L'addition de glycérol ( $1 \mathrm{mmol} / \mathrm{l})$ en présence de $\mathrm{Mg}^{2+}$ permet aux cellules de la souche ML3 de conserver $50 \%$ de viabilité après $38 \mathrm{~h}$ de privation nutritionnelle (Thomas et Batt, 1968). Strange et Dark (1965) ont observé, pour $A$ aerogenes, que des taux croissants d'oxydation du glycérol produisent une diminution de la viabilité cellulaire.

\section{Croissance cryptique}

Ce phénomène, au cours duquel des cellules se multiplient à partir des produits de dégradation libérés par les organismes morts, se produit en culture pure de certaines bactéries qui ont des exigences nutritionnelles simples (Harrison, 1960; Postgate, 1976). Dans le cas de $L$ c lactis, les cellules sont lentement dégradées par leurs propres enzymes, mais il n'y a pas de croissance cryptique puisque les produits qui en résultent, sucres de la paroi cellulaire et ARN, ne sont pas catabolisés par les cellules survivantes (Thomas et 
Batt, 1968, 1969a). Ils représentent par contre une source d'énergie et de carbone pour d'autres bactéries de la microflore du fromage telles que $L b$ plantarum, $L b$ casei, $L$ b brevis ou Pediococcus pentosaceus (Thomas, 1987).

\section{Caractéristiques de croissance des cellules survivantes}

Les cellules de $L c$ lactis ML3 survivantes présentent une croissance avec une phase de latence beaucoup plus longue que celle des cellules non soumises à des conditions de privation (Thomas et Batt, 1968). $\mathrm{Au}$ lieu des colonies circulaires typiques, de nombreux organismes produisent des colonies filamenteuses dont les cellules montrent toujours un retard important à la division. Cette latence suggère que la restauration de l'intégrité cellulaire initiale, après transfert dans un milieu optimum, est un processus long ou que le substrat influence le métabolisme de l'organisme qui se comporte alors comme une bactérie soumise à une répression de la synthèse enzymatique (Postgate et Hunter, 1964).

\section{Conclusion}

Plusieurs facteurs inhérents à la souche, aux conditions de culture ou de privation nutritionnelle peuvent influencer la viabilité des organismes. Dans ce contexte, la viabilité des bactéries étudiées en laboratoire n'est pas forcément étroitement corrélée avec les limites de survie dans l'environnement naturel. Toutefois, quand plusieurs souches de Lactococcus sont étudiées conjointement, leur capacité à survivre est très proche, mais toutefois beaucoup plus courte que celle d'autres espèces bactériennes telles Arthrobacter s p qui con- serve $50 \%$ de viabilité après 1680 h de privation nutritionnelle (Ensign, 1970).

Chez Nocardia corallina, le taux de consommation d'oxygène, la dégradation des glucides et le rejet d'ammoniaque dans le milieu extérieur sont approximativement 5 fois plus lents (Robertson et Batt, 1973) que chez $E$ coli (Dawes et Ribbons, 1965), tandis que la capacité à survivre de $N$ corallina est également largement supérieure ( $480 \mathrm{~h}$ contre $36 \mathrm{~h}$ ).

La diminution de la viabilité est notamment corrélée au taux de dégradation des macromolécules pour chaque organisme au cours de la privation nutritionnelle.

\section{ÉTUDE DU POIDS SEC ET DE LA TURBIDITÉ}

L'étude du poids sec au cours du séjour de cellules de $L c$ lactis en solution tampon est uniquement rapportée par Thomas et Batt (1969a). Après $28 \mathrm{~h}$ de privation nutritionnelle, le poids sec des cellules de $L c$ lactis ML3 a diminué de $26 \%$ et cette diminution reflète la dégradation concomitante des constituants cellulaires.

Le suivi de la turbidité des cultures ou des suspensions cellulaires permet d'évaluer l'activité autolytique de la souche qui peut être exprimée par :

- le calcul du pourcentage de diminution de la turbidité par rapport à l'instant initial dans le cas de cellules mises en suspension en solution tampon (McDonald, 1971; Mou et al, 1976; Niskasaari, 1989) ou par rapport à la DO maximale dans le cas de cultures en milieu non renouvelé (Vegarud et al, 1983; Langsrud et al, 1987);

- le calcul du rapport de la DO la plus basse à la DO la plus forte (Langsrud et al, 1978; Vegarud et Langsrud, 1982). 


\section{DÉGRADATION ET LIBÉRATION DES CONSTITUANTS CELLULAIRES}

La perte progressive des constituants cellulaires, qui survient lorsque les bactéries sont placées en conditions de privation nutritionnelle, résulte d'un déséquilibre entre les réactions d'anabolisme et de catabolisme. Elle peut également provenir d'autres processus tels que la fuite ou la sécrétion de composés cellulaires. Une synthèse de novo ou un turn-over des polymères cellulaires peut se dérouler, mais le métabolisme, essentiellement catabolique, peut à plus ou moins long terme entraîner la mort des organismes. Ces activités métaboliques des bactéries placées en conditions de privation nutritionnelle sont habituellement regroupées sous le terme de "métabolisme endogène" (Dawes et Ribbons, 1962; Dawes, 1976).

Tous les composés de la cellule font généralement l'objet d'une dégradation chez les organismes, c'est-à-dire le matériel de réserve, les glucides, les lipides, les protéines, les acides aminés et les acides nucléiques.

Les taux et l'ordre de la dégradation des substrats ainsi que le schéma général du métabolisme endogène varient selon :

- les organismes;

- les conditions de croissance et donc l'état physiologique des organismes;

- les conditions physico-chimiques de l'environnement de privation nutritionnelle.

Les changements de la composition des bactéries placées en conditions de privation nutritionnelle accompagnant une perte de la viabilité sont relativement bien décrits. Toutefois, la plupart des études concernent des bactéries Gram-, susceptibles d'accumuler des réserves telles que le glycogène ou le polyhydroxybutyrate. Comparativement, peu de travaux concernent les bactéries du genre Lactococcus.

\section{Polymères de réserve}

Des analyses chimiques pratiquées sur des cellules de Lc lactis ML3 placées en conditions de privation nutritionnelle, ainsi que des observations microscopiques n'ont pas révélé la présence de glycogène, de polyhydroxybutyrate (Thomas et Batt, 1969a) ou de granules de polyphosphate (Thomas et al, 1969).

\section{Glucides}

Les cellules de $L$ c lactis ML3 prélevées après croissance en milieu limité en lactose et transférées en solution tampon ne présentent pas de dégradation appréciable des hexoses cellulaires totaux (Thomas et Batt, 1968). Le dosage des sucres aminés du peptidoglycane et de leurs dérivés a été parfois effectué afin de caractériser l'autolyse des cellules de $L$ c lactis subsp cremoris (Mou et al, 1976; Niskasaari, 1989).

\section{Lipides}

Les cellules de Lc lactis ML3 en culture contiennent $3,8 \%$ de lipides (exprimés par rapport au poids sec). Une perte de $10 \%$ de ces lipides survient après une période de $55 \mathrm{~h}$ de privation nutritionnelle (Thomas et Batt, 1969a).

La fraction lipidique polaire ne représente alors plus que $70 \%$ de la fraction lipidique totale, contre $85 \%$ initialement. Cette diminution peut être mise en évidence par l'augmentation des acides gras libres au cours de la privation nutritionnelle.

La fraction lipidique neutre augmente de 12 à $30 \%$ des lipides totaux. La composition de la fraction acides gras libres des fractions lipidiques neutre et polaire varie. La quantité relative d'acide hexadécanoï- 
que diminue dans la fraction lipidique neutre tandis que l'acide tétradécanoïque augmente. L'acide lactobacillique (acide 2hexylcyclopropanedécanoïque) augmente dans chacune des fractions lipidiques avec une diminution parallèle de son précurseur immédiat, l'acide cis-vaccénique.

D'après Guckert et al (1986), l'utilisation des acides gras cismonoéiques au cours de la privation nutritionnelle semble préférentielle. Les capacités à synthétiser des acides transmonoéiques qui ne sont généralement pas facilement métabolisés par les bactéries, ou à modifier les acides cismonoéiques en leurs dérivés cyclopropyl, seraient des mécanismes de survie qui aideraient à maintenir une membrane fonctionnelle, bien que structurellement altérée, au cours de la privation nutritionnelle.

Une faible quantité d'acides gras volatils ( $0,6 \mathrm{mEq} / \mathrm{g}$ de poids sec) est libérée en $24 \mathrm{~h}$ dans la solution tampon par les cellules de $L c$ lactis. L'origine de ces éléments n'a pas été déterminée.

Les lipides polaires, dont les phospholipides, constituent la fraction lipidique principale des cellules de $L c$ lactis ML3. Ces phospholipides sont connus pour avoir un rôle structural important dans les membranes bactériennes; en conséquence, toute variation concernant les phospholipides est susceptible d'interférer avec l'intégrité des barrières cellulaires (Kates, 1964).

\section{Acides nucléiques}

La dégradation de I'ADN intracellulaire a été observée pour les cellules de $L$ c lactis subsp cremoris C1 (Krishna et Dutta, 1976) et H-61 (Ohmiya et Sato, 1969), Lc lactis subsp lactis CNRZ 141 (ML3) et CNRZ 125 (Foucaud et al, soumis pour publication) placées en conditions de privation nutritionnelle ainsi que pour diverses souches de lactocoques en milieu M17 (Langsrud et al, 1987). Dans cette étude, les auteurs ont observé que la quantité d'ADN cellulaire diminue parallèlement à la turbidité de la suspension; dans ces conditions, la mesure des changements concernant I'ADN intracellulaire se révèle être un bon marqueur du phénomène de lyse (Langsrud et al, 1987).

En revanche, aucun changement de la quantité d'ADN cellulaire n'a été rapporté au cours de la maturation d'un caillé réalisé avec des cellules de $L c$ lactis subsp cremoris (Ohmiya et Sato, 1970). Dans le cas de cellules de $L c$ lactis ML3, les auteurs rapportent alternativement une perte d'ADN corrélée à la viabilité cellulaire (Thomas et al, 1969) et une constance de la quantité intracellulaire d'ADN, initialement de $3,7 \%$ du poids sec initial (Thomas et Batt, 1969a). La densité cellulaire 20 fois plus grande dans le deuxième cas n'est pas invoquée et seule la variabilité des résultats semble en cause faute d'une autre explication.

En absence de $\mathrm{Mg}^{2+}$, l'ARN des cellules de $L$ c lactis ML3 placées en conditions de privation nutritionnelle, est rapidement dégradé. Les organismes, contenant initialement $20,8 \%$ d'ARN (calculés par rapport au poids sec initial) ne présentent plus que $5,5 \%$ d'ARN après $28 \mathrm{~h}$ (Thomas et Batt, 1969a). En présence de $\mathrm{Mg}^{2+}$, la dégradation de l'ARN, environ 2 fois moins importante, ne survient qu'après une phase de latence relativement longue (Thomas et Batt, 1969a).

Une quantité substantielle de matériel soluble, ayant une absorption maximale à $257 \mathrm{~nm}$, est rejetée par les organismes (Thomas et Batt, 1969a). Cela peut être corrélé à la dégradation des acides nucléiques et à la perte des fragments correspondants absorbant en UV (purines et pyrimidines). 


\section{Protéines et acides aminés}

Au cours du séjour des cellules de $L$ c lactis ML3 en solution tampon, les protéines qui représentent initialement $48 \%$ du poids sec bactérien, sont faiblement dégradées : $15 \%$ de la quantité initiale de protéines après $28 \mathrm{~h}$ (Thomas et Batt, 1969a).

La perte des protéines par les cellules de $L$ c lactis ML3 paraît ne résulter que de réactions d'hydrolyse avec libération des produits de dégradation dans le milieu extérieur (Thomas et Batt, 1969a). Bien que la quantité globale d'acide aspartique diminue au cours de l'incubation des cellules, il n'est pas évident que les composants du pool d'acides aminés libres de $L c$ lactis fassent l'objet de réactions anaboliques (Thomas et Batt, 1969a).

Le pool des acides aminés intracellulaires des cellules de $L$ c lactis ML3 qui représente initialement $3,3 \%$ du poids sec initial, est rapidement dégradé; après $28 \mathrm{~h}$ de privation nutritionnelle, il ne représente plus que $24 \%$ de sa valeur initiale (Thomas et Batt, 1969a). Parallèlement, des acides aminés apparaissent dans le milieu extérieur. L'ornithine et l'arginine ne sont jamais décelés, contrairement à l'acide glutamique, l'alanine et la lysine (Thomas et Batt, 1969a). Après $28 \mathrm{~h}$ de privation nutritionnelle, on constate une nette augmentation de la quantité totale d'acides aminés libres, qui traduit une dégradation des protéines en plus de la libération d'acides aminés libres (Thomas et Batt, 1969a). Cette augmentation des concentrations d'acides aminés correspondrait à une dégradation de $5 \%$ des protéines bactériennes (Thomas et Batt, 1969a).

\section{Activités enzymatiques}

La libération, dans le milieu extérieur, d'une enzyme intracellulaire, telle que la $\beta$ - galactosidase ( $\beta$-gal) a été utilisée comme index de la lyse de cellules de $B$ subtilis (Pollock, 1961). Dans le cas de Lc lactis ML3, aucune activité $\beta$-gal n'est décelée dans le surnageant de suspension des cellules (Thomas et al, 1969). Citti et al (1965) rapportent que l'activité $\beta$-gal libérée à la suite d'un stress par 5 souches de $L c$ lactis est très instable. Cette observation pourrait expliquer la perte rapide d'activité de cette enzyme lorsqu'elle est libérée au cours de l'incubation des cellules en solution tampon.

Le dosage dans le milieu extérieur de la lactate déshydrogénase (LDH), à la suite de la rupture de la paroi et de la membrane cellulaire (Ohmiya et Sato, 1969), montre des variations selon les souches testées (Langsrud et al, 1978, 1987; Vegarud et Langsrud, 1987). Cette activité enzymatique paraît instable à certains auteurs (Thomas et al, 1969) mais se révèle être un bon marqueur de lyse pour d'autres (Langsrud et al, 1987).

L'activité prolyliminopeptidase (PIP) a fait plus récemment l'objet de dosages dans le cas de cellules de Lc lactis étudiées en conditions de privation nutritionnelle (Langsrud et al, 1978, 1987; Vegarud et Langsrud, 1982, 1987). Cette investigation, motivée par le rôle des lactocoques dans l'hydrolyse des caséines et par la quantité importante de proline dans ces protéines, a montré que l'activité PIP serait le fait de plusieurs activités d'hydrolyse des peptides qui varieraient en proportions différentes au cours de la croissance (Langsrud et al, 1987). D'autre part, il est difficile de préciser dans quelle mesure et à quel stade de l'incubation l'activité PIP est d'origine intracellulaire ou liée à la membrane. La détermination de l'activité PIP en qualité d'indicateur d'autolyse demanderait à être réévaluée (Langsrud et al, 1987). 


\section{Conclusion}

La dégradation des constituants intracellulaires et la perte concomitante de composés sont des phénomènes complexes et susceptibles de varier sous l'influence de nombreux facteurs. II est, cependant, difficile d'associer la perte de viabilité ou de poids sec à la dégradation ou à la libération d'un composé spécifique mais la participation du système autolytique dans ce processus est importante.

\section{SYSTĖME AUTOLYTIQUE}

L'autolyse se caractérise par une rupture de la paroi bactérienne dont sont responsables une ou des autolysine(s).

Malgré l'importance du système responsable de l'autolyse chez les bactéries du genre Lactococcus à l'image de ce qu'il en est chez d'autres bactéries, peu de travaux ont porté sur sa mise en évidence et sa caractérisation (Mou et al, 1976; Niskasaari, 1989).

L'autolysine de Lc lactis subsp cremoris est localisée dans la paroi bactérienne. Ce système autolytique, étudié en solution tampon, présente les mêmes conditions de $\mathrm{pH}$, de concentration et de température que les organismes entiers pour développer une activité optimale. II ne montre pas d'activité amidase ou endopeptidase. II a la spécificité d'une endo-N-acétylmuramidase responsable de l'hydrolyse de la liaison entre la $\mathrm{N}$-acétylglucosamine et l'acide $\mathrm{N}$ acétylmuramique dans la paroi.

\section{MODIFICATIONS MÉTABOLIQUES ASSOCIÉES AUX CONDITIONS DE PRIVATION NUTRITIONNELLE}

Les lactocoques qui fermentent de façon homolactique le glucose et le lactose, peu- vent développer un métabolisme hétérofermentaire lors de culture en présence de concentrations limitantes de lactose ou de glucose (Thomas, 1976; Thomas et al, 1979). Les produits formés alors sont le formate, l'acétate et l'éthanol.

Une régulation fine du métabolisme glucidique est susceptible de s'exercer au niveau de la modulation des activités enzymatiques, en réponse aux fluctuations des concentrations des effecteurs intracellulaires [fructose-1,6-diphosphate (FDP), phosphoénolpyruvate (PEP), phosphate inorganique $\left.\left(P_{i}\right)\right]$.

Le potentiel PEP consiste en 3 intermédiaires (PEP, 3-phosphoglycérate, 2phosphoglycérate) de la glycolyse qui représentent au total environ $40 \mathrm{mmol} / \mathrm{l}$ chez les cellules en privation nutritionnelle, soit 4 fois plus que chez les cellules en croissance (Thompson, 1978, 1987). Ce potentiel apparaît lors de la transition vers un état de privation nutritionnelle et peut être maintenu pendant plusieurs heures chez Lc lactis subsp lactis (Thompson et Thomas, 1977; Mason et al, 1981) ou seulement pendant quelques minutes chez certaines souches de $L \quad c$ lactis subsp cremoris (Otto et al, 1985). L'explication biochimique de la formation de ce pool endogène a donné lieu à des controverses (Thompson et Thomas, 1977; Mason et al, 1981; Otto et al, 1985; Lohmeier-Vogel et al, 1986). Cependant, des données obtenues par analyse enzymatique (Thompson et Thomas, 1977), par ${ }^{14} \mathrm{C}$-fluorographie (Thompson et Torchia, 1984) et par spectroscopie RMN du ${ }^{31} \mathrm{P}$ in vivo (Thompson et Torchia, 1984; Lohmeier-Vogel et al, 1986) suggèrent que l'existence du potentiel PEP résulte de l'inactivation de la pyruvate kinase $(\mathrm{PK})$ par :

- épuisement des stimulateurs de l'ensyme allostérique (FDP, par exemple), (Collins et Thomas, 1974; Thompson, 1978); 
- augmentation marquée de la concentration du $\mathrm{P}_{\mathrm{i}}$, inhibiteur potentiel de la PK in vitro (Collins et Thomas, 1974; Crow et Pritchard, 1976).

La présence de ce potentiel PEP chez les bactéries étudiées en conditions de privation nutritionnelle présente une grande importance physiologique :

- la lente utilisation du PEP par la PK peut fournir l'énergie de maintenance nécessaire pour ces organismes au cours de la privation nutritionnelle (Thompson et Thomas, 1977; Otto et al, 1985);

- les cellules placées en conditions de privation nutritionnelle seront capables d'effectuer le transport immédiat du lactose ou d'autres sucres concernés par le système PTS lorsque ces sucres seront à nouveau disponibles (Thompson, 1987).

Lors de la suppression de la source glucidique dans le milieu de culture, les cellules de $L c$ lactis subsp cremoris restent viables pendant $20 \mathrm{~h}$ tandis que la force protomotrice (pmf) s'annule progressivement après 60 à $90 \mathrm{~min}$ de privation nutritionnelle (Otto et al, 1983, 1984; Poolman et al, 1987a). Cette diminution est associée à un épuisement du pool intracellulaire de sucres phosphatés et à une décroissance du niveau d'ATP qui reste cependant suffisant pour générer un gradient de $\mathrm{pH}$ (Konings et al, 1989). Cependant, au cours de la privation nutritionnelle, la présence des intermédiaires riches en énergie ne semble pas cruciale pour la survie (Poolman et al, 1987a).

L'analyse du pool intracellulaire d'acides aminés indique que les cellules de $L$ c lactis subsp cremoris $\mathrm{Wg}_{2}$ sont capables, en absence de source d'énergie, de maintenir des gradients élevés de concentration pour les acides glutamique et aspartique, l'alanine et la glycine, indiquant que les systèmes de transport correspondants sont inactifs chez ces cellules et/ou ne facilitent pas leur sortie.
L'importance du pool d'acide glutamique (> $100 \mathrm{mmol} / \mathrm{l}$ ) suggère que ce soluté contribue à la maintenance de la turgescence cellulaire et que cette accumulation a pour support énergétique I'ATP ou mieux un intermédiaire phosphorylé riche en énergie et non la pmf (Poolman 1987a, 1987b, 1987c).

\section{MODIFICATIONS GÉNOTYPIQUES ASSOCIÉES AUX CONDITIONS DE PRIVATION NUTRITIONNELLE}

Le séjour des cellules de LC lactis (souches C2 et ML3) pendant une période longue $\left(96 \mathrm{~h}\right.$ ) à $32^{\circ} \mathrm{C}$ en milieu M 17 non tamponné par suppression du $\beta$ glycérophosphate de sodium induit au hasard la perte de plasmides de tailles différentes. Si le processus de croissance dans ce milieu M 17 est répété, la majeure partie des plasmides est alors perdue (Sinha, 1989a).

Le mode d'élimination des plasmides par incubation prolongée des souches en milieu M 17 contenant du lactose en quantité faible $(5 \mathrm{~g} / \mathrm{l})$, semble similaire au phénomène décrit chez $E$ coli. Des souches d'E coli exigeant la thymine, perdent leurs plasmides lors de la croissance dans un milieu dont la concentration en thymine est limitante (Clowes et al, 1965) ou dépourvu de thymine (Pinney et Smith, 1971).

Lorsque des cellules de $L$ c lactis $\mathrm{C} 2$, provenant d'une culture en milieu M 17 , sont transférées en milieu $M 17$ dépourvu de lactose et incubées de façon prolongée à $32{ }^{\circ} \mathrm{C}$, la majorité des cellules viables présente un phénotype Lac-Prt+ (Sinha, 1989b).

Le transfert en solution tampon de cellules de $L$ c lactis subsp lactis CNRZ 125, préalablement cultivées en milieu complexe, favorise l'obtention de variants qui forment des colonies de faible diamètre 
sur milieu M 17 gélosé (Foucaud et al, 1990). La proportion de variants de la souche CNRZ 125 dépend des conditions de culture et de séjour en solution tampon. Les variants sont incapables de métaboliser le lactose et ont perdu sélectivement un plasmide de 32,3 MDa. Les mêmes conditions ne permettent pas l'obtention de variants lac- pour 4 autres souches de $L C$ lactis testées.

\section{CONCLUSION}

Les données de la littérature relatives à la physiologie des bactéries du genre Lactococcus en conditions de privation nutritionnelle conduisent à plusieurs remarques :

- les phénomènes survenant au cours de ce processus sont nombreux et intéressent la structure comme la physiologie cellulaire;

- l'initiation et le développement des phénomènes sont largement susceptibles d'être influencés par des facteurs liés aux conditions de culture ou de privation nutritionnelle mais également aux caractères de la souche étudiée;

- il n'est pas possible d'associer la perte de viabilité d'une bactérie à celle d'un constituant cellulaire particulier mais plutôt au taux de dégradation des macromolécules de structure comme de fonction;

- les conditions de privation nutritionnelle sont susceptibles de faire ressortir le comportement particulier de souches de $L C$ lactis : sensibilité aux conditions d'environnement ( $\mathrm{NaCl}$, par exemple), activité autolytique plus ou moins développée, perte de plasmide(s) conduisant à l'obtention de souches modifiées stables. Comme nous l'avons souligné, ces particularités peuvent intéresser, d'une part l'industrie laitière (salage, obtention et maturation du caillé, métabolisme protéique, production d'arômes) et d'autre part les laboratoires de recherches (souches phénotypiquement modifiées d'obtention aisée).

Bien que la physiologie des bactéries du genre Lactococcus en conditions de privation nutritionnelle soit relativement déjà bien étudiée, il serait intéressant de développer certains points suggérés par d'autres études :

- la synthèse de protéines particulières susceptibles de jouer un rôle dans la viabilité bactérienne a été notamment mise en évidence chez $E$ coli (Groat et Matin, 1986; Groat et al, 1986);

- l'accumulation de guanosine polyphosphate en réponse à la privation nutritionnelle a été décrite pour Streptococcus faecium, $S$ pyogenes et $S$ rattus (McDowell et al, 1988);

- l'induction du phénomène de lyse par les acides gras pourrait apporter des résultats supplémentaires si l'on considère leur utilisation dans le cas de $B$ subtilis, par exemple (Carson et Daneo-Moore, 1980);

- la composition du peptidoglycane d'E coli est influencée par le taux de croissance des cellules (Driehuis et Wouters, 1987);

- d'autres stratégies ont été envisagées en réponse à la limitation nutritionnelle, notamment celles concernant les systèmes de transport des nutriments (Harder et Dijkhuizen, 1983). II serait, dans cette perspective, intéressant de considérer plus amplement les systèmes de transport des peptides et acides aminés par les lactocoques;

- enfin, l'étude de la physiologie des bactéries en conditions de privation nutritionnelle pourrait s'étendre à d'autres genres bactériens concernés par l'appellation "bactéries lactiques" pour lesquels peu ( $S$ thermophilus, Sandholm et Sarimo, 1981) ou pas (Leuconostoc sp) de travaux ont été effectués ou pour lesquels des études 
plus précises n'ont été reprises que récemment ( $L b$ helveticus, Lortal et al, 1989).

\section{RÉFÉRENCES}

Abelson PH, Aldous E (1950) lon antagonisms in microorganisms: interference of normal magnesium metabolism by nickel, cobalt, cadmium, zinc, and manganese. $J$ Bacteriol $60,401-413$

Asbell MA, Eagon RG (1966) Role of multivalent cations in the organization, structure and assembly of the cell wall of Pseudomonas aeruginosa. J Bacteriol 92, 380-387

Bie R, Sjöström G (1975) Autolytic properties of some lactic acid bacteria used in cheese production. Part II. Experiments with fluid substrates and cheese. Milchwissenschaft 30 , 739-747

Bridson EY, Brecker A (1970) Design and formulation of microbial culture media. In: Methods in microbiology (JR Norris, DW Ribbons, eds). Academic Press, London, New York, 229-295

Carson DD, Daneo-Moore L (1980) Effects of fatty acids on lysis of Streptococcus faecalis. $J$ Bacteriol 141, 1122-1126

Citti JE, Sandine WE, Elliker PR (1965) Bgalactosidase of Streptococcus lactis. J Bacteriol 89, 937-942

Clowes RC, Moody EEM, Pritchard RH (1965) The elimination of extrachromosomal elements in thymineless strains. Genet Res Comb 6, 147-152

Cogan TM, Daly C (1987) Cheese starter cultures. In: Cheese. Chemistry, Physics and Microbiology. Vol 1: General Aspects (FP Fox, ed). Elsevier Applied Science Publishers, 179-249

Collins LB, Thomas TD (1974) Pyruvate kinase of Streptococcus lactis. J Bacteriol 144, 683691

Cornett JB, Johnson CA, Shockman GD (1979) Release of autolytic enzyme from Streptococcus faecium cell walls by treatment with dilute alcali. J Bacteriol 138, 699-704

Crow VL, Pritchard GG (1976) Purification and properties of pyruvate kinase from Strepto- coccus lactis. Biochim Biophys Acta 483, 90101

Dawes EA (1976) Endogenous metabolism and the survival of starved prokaryotes. In: The survival of vegetative microbes (TRG Gray and JR Postgate, eds). Cambridge University Press, 19-53

Dawes EA, Ribbons DW (1962) The endogenous metabolism of microorganisms. Annu Rev Microbiol 16, 241-264

Dawes EA, Ribbons DW (1964) Some aspects of the endogenous metabolism of bacteria. Bacteriol Rev 28, 126-149

Dawes EA, Ribbons DW (1965) Studies on the endogenous metabolism of Escherichia coli. Biochem J 95, 322-343

Driehuis F, Wouters JTM (1987) Effect of growth rate and cell shape on the peptydoglycan composition of Escherichia coli. $J$ Bacteriol $169,97-101$

Elmros T, Burman LG, Bloom GD (1976) Autolysis of Neisseria gonorrhoeae. J Bacteriol 126, 969-976

Ensign JC (1970) Long-term starvation survival of rod and spherical cells of Arthrobacter crystallopoietes. J Bacteriol 103, 569-577

Foucaud C, Furlan S, Winters D, Hemme D (1990) Specific loss of the plasmid encoding for lactose metabolism by Lactococcus lactis CNRZ 125. Milchwissenschaft 45 (sous presse)

Gray GW, Wilkinson SG (1965) The effect of ethylenediaminetetraacetic acid on the cell walls of Gram-negative bacteria. J Gen Microbio/ 39, 385-399

Groat RG, Matin A (1986) Synthesis of unique proteins at the onset of carbon starvation in Escherichia coli. J Ind Microbiol 1, 69-73

Groat RG, Schultz JE, Zychlinsky E, Bockman A, Matin A (1986) Starvation of proteins in Escherichia coli: kinetics of synthesis and role in starvation survival. $J$ Bacteriol 168 , 486-493

Gruckert JB, Hood MA, White DC (1986) Phospholipid ester-linked fatty acid profiles changes during nutrient deprivation of Vibriocholerae: increases in the trans/cis ratio and proportions of cyclopropane fatty acids. Appl Environ Microbiol 52, 794-801

Guinee TP, Fox FP (1987) Salt in cheese: physical, chemical and biological aspects. In: 
Cheese. Chemistry, Physics and Microbiology. Vol 1: General Aspects (FP Fox, ed). Elsevier Applied Science Publishers, 251-298

Harder W, Dijkhuizen L (1983) Physiological responses to nutrient limitation. Annu Rev Microbiol 37, 1-23

Harrison AP (1960) The response of Bacterium lactis aerogenes when held at growth temperature in the absence of nutriment: an analysis of survival curves. Proc Res Soc $B$ $152,418-428$

Hinks ET, Daneo-Moore L, Braverman S (1978) Effects of temperature on the autolytic system of Streptococcus faecalis. J Bacteriol $136,491-496$

Ito S, Kobayashi T, Osaki K, Morichi T, Saitoh M (1987) Microbial cell division and separation: effect of citrate on the growth of group $\mathrm{N}$ streptococci. Food Microstruct 6, 17-24

Kates M (1964) Bacterial lipids. In: Advances in lipid research (R Paoletti and D Kritchevsky, eds). Academic Press, 17-90

Konings WN, Poolman B, Driessen AJM (1989) Bioenergetics and solute transport in lactococci. CRC Crit Rev Microbiol 16, 419-476

Kontusaari S, Forsén R (1987) Cell surface proteins of encapsulated Streptococcus cremoris: identification and immunochemical characterization. J Appl Bacteriol 63, 133-137

Krishna BM, Dutta SM (1976) Studies on the autolytic changes in $S$ cremoris under starvation conditions. Milchwissenschaft 31, 741744

Langsrud T, Castberg HB, Landaas A (1978) Propriétés autolytiques de quelques streptocoques des levains. XX Congr Int Lait, 526527

Langsrud T, Laandas A, Castberg HB (1987) Autolytic properties of different strains of group N streptococci. Milchwissenschaft 42, 556-560

Law BA (1987) Proteolysis in relation to normal and accelerated cheese ripening. In: Cheese. Chemistry, Physics and Microbiology. Vol 1: General Aspects. (FP Fox, ed). Elsevier Appli Sci Publi, Barking, 365-394

Law BA, Sharpe ME, Reiter B (1974) The release of intracellular dipeptidase from starter streptococci during Cheddar cheese ripening. J Dairy Res 41, 137-146
Lohmeier-Vogel EM, Hahn-Hagerdahl B, Vogel J (1986) Phosphorus-31 NMR studies of glucose and glucose metabolism in Streptococcus lactis. Appl Microbiol Biotechnol 25, 4351

Lortal S, Boyaval P, Van Heijenoort J (1989) Influence de plusieurs facteurs sur l'autolyse de Lactobacillus helveticus CNRZ 414. Lait 69, 223-231

McDonald IJ (1971) Filamentous forms of Streptococcus cremoris and Streptococcus lactis. Observations on structure and susceptibility to lysis. Can J Microbiol 17, 897-902

McDowell TD, Reed KE, Hadley WM (1988) ACcumulation of $\mathrm{ppGpp}$ in three streptococci during periods of amino acids starvation. FEMS Microbiol Lett 56, 151-156

McLeod RA, Snell EE (1950) The relation of ion antagonism to the inorganic nutrition of lactic acid bacteria. J Bacterio/ 59, 783-792

Mager J (1959) The stabilizing effect of spermine and related polyamines on bacterial protoplasts. Biochim Biophys Acta 36, 529531

Martley FG (1972) The effect of cell numbers in streptococcal chains on plate-counting. $N Z J$ Dairy Sci Technol 7, 7-11

Mason CA, Hamer G, Bryers JD (1986) The death and lysis of microorganisms in environmental processes. FEMS Microbiol Rev 39, 373-401

Mason PW, Carbone DP, Cushman RA, Waggoner AS (1981) The importance of inorganic phosphate in regulation of energy metabolism of Streptococcus lactis. J Biol Chem 256, 1861-1866

Mou L, Sullivan JJ, Jago GR (1976) Autolysis of Streptococcus cremoris. J Dairy Res 43, 275282

Moustafa HH, Collins EB (1968) Role of galactose or glucose-1-phosphate in preventing the lysis of Streptococcus diacetylactis. J Bacteriol 95, 592-602

Niskasaari K (1989) Characteristics of the autolysis of variants of Lactococcus lactis subsp cremoris. J Dairy Res 56, 639-649

Ohmiya K, Sato Y (1969) Studies on the proteolytic action of dairy lactic acid bacteria. Part IX. Autolysis and proteolytic action of Streptococcus cremoris and Lactobacillus helveticus. Agric Biol Chem 33, 1628-1635 
Ohmiya K, Sato Y (1970) Studies on the proteoIytic action of dairy lactic acid bacteria. Part $X$. Autolysis of lactic acid bacteria cells in aseptic rennet curd. Agric Biol Chem 34, 457-463

Otto $R$, Ten Brink B, Velkamp H, Konings WN (1983) The relation between growth rate and electrochemical proton gradient of Streptococcus cremoris. FEMS Microbiol Lett 16, 69-74

Otto R, Klont B, Ten Brink B, Konings WN (1984) The phosphate potential, adenylate energy charge and proton motive force in growing cells of Streptococcus cremoris. Arch Microbiol 139, 338-343

Otto R, Vije T, Ten Brink B, Klont B, Konings WN (1985) Energy metabolism in Streptococcus cremoris during lactose starvation. Arch Microbiol 141, 348-352

Pinney RJ, Smith JT (1971) R-factor elimination by thymine starvation. Genet Res Comb 18, 173-177

Pirt SJ (1965) The maintenance energy of bacteria in growing cultures. Proc $A$ Soc Lond $163,224-231$

Pollock MR (1961) The measurement of the liberation of penicillinase from Bacillus subtilis. J Gen Microbio/ 26, 239-253

Poolman B, Driessen AJM, Konings WN (1987a) Regulation of solute transport in streptococci by external and internal pH values. Microbiol Rev 51, 498-508

Poolman B, Smid EJ, Konings WN (1987b) Kinetic properties of a phosphate-bond-driven system in Streptococcus lactis and Streptococcus cremoris. J Bacteriol 169, 2755-2761

Poolman B, Smid EJ, Veldkamp H, Konings WN (1987c) Bioenergetic consequence of lactose starvation for continuously cultured Streptococcus cremoris. J Bacteriol 169, 1460-1468

Postgate JR (1976) Death in macrobes and microbes. In: The survival of vegetative microbes (TRG Gray and JR Postgate, eds). Cambridge University Press, 1-18

Postgate JR, Hunter JR (1962) The survival of starved bacteria. J Gen Microbiol 29, 233263

Postgate JR, Hunter JR (1964) Accelerated death of Aerobacter aerogenes starved in the presence of growth-limiting substrates. $J$ Gen Microbiol 34, 459-473
Postgate JR, Crumpton JE, Hunter JR (1961) The measurement of bacterial viabilities by slide culture. J Gen Microbiol 24, 15-24

Prasad C, Freese E (1974) Cell lysis of Bacillus subtilis caused by intracellular accumulation of glucose-1-phosphate. J Bacteriol 118, 1111-1122

Robertson JG, Batt RD (1973) Survival of Nocardia corallina and degradation of constituents during starvation. J Gen Microbiol 78, 109-117

Roznak DB, Colwell RR (1987) Survival strategies of bacteria in the natural environment. Microbiol Rev 51, 365-379

Sandholm E, Sarimo SS (1981) Autolysis of Streptococcus thermophilus. FEMS Microbiol Lett 11, 125-129

Sinha RP (1989a) A new simple method of curing plasmids in lactic streptococci (Streptococcus cremoris; Streptococcus lactis, plasmids). FEMS Microbiol Lett 57, 349-352

Sinha RP (1989b) Effect of starvation on the loss of plasmids in Lactococcus lactis C2. ADSANASAS Abstracts, Teaming up for animal agriculture, 277

Strange RE, Dark FA (1965) Substrate accelerated death of Aerobacter aerogenes. $J$ Gen Microbiol 39, 215-228

Sundman V (1957) On the protein character of a slime produced by Streptococcus cremoris in finnish ropy sour milk. Acta Chem Scand 7, 558-559

Svarachorn A, Shinmyo A, Tsuchido T, Takano $M$ (1989) Autolysis of Bacillus subtilis induced by monovalent cations. Appl Microbiol Biotechnol 30, 299-304

Tabor CW (1962) Stabilization of protoplasts and spheroplasts by spermine and other polyamines. J Bacteriol 83, 1101-1111

Terracciano JS, Rapaport E, Kashket ER (1988) Stress- and growth phase-associated proteins of Clostridium acetobutylicum. Appl Environ Microbiol 54, 1989-1995

Thomas TD (1976) Regulation of lactose fermentation in group N streptococci. Appl Environ Microbiol 32, 474-478

Thomas TD (1987) Cannibalism among bacteria found in cheese. N Z J Dairy Sci Technol 22, 215-219 
Thomas TD, Batt RD (1968) Survival of Streptococcus lactis in starvation conditions. J Gen Microbiol 50, 367-382

Thomas TD, Batt RD (1969a) Degradation of cell constituents by starved Streptococcus lactis. J Gen Microbiol 58, 347-362

Thomas TD, Batt RD (1969b) Synthesis of protein and ribonucleic acid by starved Streptococcus lactis in relation to survival. J Gen Microbiol 58, 363-369

Thomas TD, Batt RD (1969c) Metabolism of exogenous arginine and glucose by starved Streptococcus lactis in relation to survival. $J$ Gen Microbiol 58, 371-380

Thomas TD, Pritchard GG (1987) Proteolytic enzymes of dairy starter cultures. FEMS Microbiol Rev 46, 245-268

Thomas TD, Ellwood DC, Longyear VM (1979) Changes from homo- to heterolactic fermentation by Streptococcus lactis resulting from glucose limitation in anaerobic chemostat cultures. J Bacteriol 138, 109-117

Thomas TD, Lyttleton P, Williamson KI, Batt RD (1969) Changes in permeability and ultrastructure of starved Streptococcus lactis in relation to survival. J Gen Microbiol 58, 381390

Thompson J (1978) In vivo regulation of glycolysis and characterization of sugar/ phosphotransferase systems in Streptococcus lactis. J Bacteriol 136, 465-476

Thompson J (1987) Sugar transport in the lactic acid bacteria. In: Sugar transport and metabolism in Gram-positive bacteria (J Reizer and A Peterkofsky, eds). Ellis Horwood Ltd, Chichester, 13-38

Thompson J, Thomas TD (1977) Phosphoenolpyruvate and 2-phosphoglycerate: endogenous energy source(s) for sugar accumulation by starved cells of Streptococcus lactis. $J$ Bacteriol 130, 583-595

Thompson J, Thorchia DA (1984) Use of ${ }^{31} \mathrm{P}$ nuclear magnetic resonance spectroscopy and ${ }^{14} \mathrm{C}$ fluorography in studies of glycolysis and regulation of pyruvate kinase in Streptococcus lactis. J Bacteriol 158, 791-800

Tipper DJ (1969) Mechanism of autolysis of isolated cell walls of Staphylococcus aureus. $J$ bacteriol 97, 837-847

Vegarud G, Langsrud T (1982) Growth and autolysis of Streptococcus diacetylactis ATCC 15346 and Streptococcus lactis 2. XXI Int Dairy Congr, 383-384

Vegarud G, Langsrud T (1987) Studies on the autolytic properties of different strains of group N streptococci. FEMS Microbiol Rev 46,59

Vegarud G, Castberg HB, Langsrud T (1983) Autolysis of group $\mathrm{N}$ streptococci. Effects of media composition modifications and temperature. J Dairy Sci 66, 2294-2302

Wade HE (1961) The autodegradation of ribonucleoprotein in Escherichia coli. Biochem J 78, 457-472

Young FE (1966) Autolytic enzyme associated with cell walls of Bacillus subtilis. J Biol Chem 241, 3462-3467 